\title{
Phantom Validation for Ultrasound to Statistical Shape Model Registration of Human Pelvis
}

\author{
Sahar Ghanavati $^{a}$, Parvin Mousavi* ${ }^{*}$, Gabor Fichtinger $^{b}$ and Purang Abolmaesumi ${ }^{c}$ \\ ${ }^{a}$ Department of Electrical and Computer Engineering, Queen's University, Canada; \\ ${ }^{b}$ School of Computing, Queen's University, Canada; \\ ${ }^{c}$ Department of Electrical and Computer Engineering, University of British Columbia, Canada.
}

\begin{abstract}
Total Hip Replacement (THR) has become a common surgical procedure in recent years, as a result of increasing aging population with osteoarthritis of the hip joint. Localization of the pelvic anatomical coordinate system $(\mathrm{PaCS})$ is a critical step in accurate placement of the femur prosthesis in the acetabulum in THR. Intra-operative ultrasound (US) imaging can provide a radiation-free navigation system for localization of the PaCS. However, US images are noisy and cannot provide any anatomical information beneath the bone surface due to the total reflection of US beam at the bone-soft tissue interface. A solution to this problem is to fuse intra-operative US with pre-operative imaging or a statistical shape model (SSM) of the pelvis. Here, we propose a multi-slice to volume intensity-based registration of the pelvic SSM to a sparse set of 2D US images in order to localize the $\mathrm{PaCS}$ in the US. In this registration technique, a set of 2D slices are extracted from a pelvic SSM using the approximate location and orientation of their corresponding 2D US images. During the registration, the comparison between the SSM slices and the US images is made using an ultrasound simulation technique and a correlation-based similarity metric. We demonstrate the feasibility of our proposed approach in localizing the PaCS on five patient-based phantoms. These results indicate the necessity of including pubic symphysis landmarks in the 2D US slices in order to have a precise estimation of the PaCS.
\end{abstract}

Keywords: Ultrasound, Statistical Shape Model, Pelvic anatomical Coordinate System, Deformable registration, Total Hip Replacement, Pelvis.

\section{INTRODUCTION}

Total Hip Replacement (THR) is a common surgical procedure that is used to treat patients with osteoarthritis in the hip joint. Misalignment of the acetabular component in the pelvis in this procedure can cause dislocation of the hip joint, ${ }^{1}$ and a revision operation would be required to correct the alignment of the acetabular component. ${ }^{2}$ The precise localization of Pelvic anatomical Coordinate System (PaCS), as a reference frame for assessing the cup and femoral prosthesis orientation in the pelvis, can decrease the chances of post-operative complications. ${ }^{3}$ PaCS is defined based on four anatomical landmarks on the pelvis: ${ }^{4}$ the left and right maximally anterior iliac crest points, and the left and right maximally anterior pubis symphysis points. To localize these anatomical landmarks, a number of US-based navigation systems have been proposed. Chan et al. ${ }^{5}$ presented a US-based SSM instantiation using Iterative Closest Point (ICP) registration for bony structures. Foroughi et al. ${ }^{6}$ proposed to register a pelvic SSM to the segmented bone surface from the US images using ICP. Barratt et al. ${ }^{7}$ presented a surface-to-surface registration method to instantiate a model of the femur and pelvis using a cloud of surface points segmented from the bone in 3D US. Schumann et al. ${ }^{8}$ proposed to use local patch-SSMs created on the left and right iliac crests and the pubis symphysis, for localization of the pelvic anatomical landmarks from US images. All these approaches require the segmentation of the bone surface from US images, which is not a trivial task and would introduce a segmentation error in the registration process.

In our previous work, we proposed a two-step multi-slice to volume registration of SSM to sparse 2D US images of the pelvis, using an US simulation technique that eliminates the need for US segmentation. ${ }^{9}$ We examined our method on two male cadaver data collected at the Johns Hopkins University. The US data only

\footnotetext{
*Send correspondence to: E-mail: pmousavi@cs.queensu.ca, Telephone: 16135336070
}

\footnotetext{
Medical Imaging 2011: Visualization, Image-Guided Procedures, and Modeling,

edited by Kenneth H. Wong, David R. Holmes III, Proc. of SPIE Vol. 7964, 79642U

(c) 2011 SPIE · CCC code: 1605-7422/11/\$18 - doi: 10.1117/12.876998
}

Proc. of SPIE Vol. 796479642 U-1 
contained the two iliac crests, which resulted in high rotation error in localization of the PaCS due to missing anatomical information of the pubic symphysis.

In this work, we improve our registration method so that instead of sequentially optimizing the rigid and SSM weighting parameters, ${ }^{9}$ we calculate these parameters simultaneously. We also validate our method on five patient-based phantoms, where in addition to the US data collected from the two iliac crests, we include the US data from the pubic symphysis as described in Section 3.1. The validation results demonstrate the importance of including US images of the pubic symphysis on precise localization of PaCS.

\section{METHODOLOGY}

\subsection{Statistical Shape Model of the Pelvis}

The SSM we have used in this work was constructed by Chintalapani et al. ${ }^{10}$ at the Johns Hopkins University, MD, USA, using $110 \mathrm{CTs}$ of healthy male patients. An instance of the SSM is created to reproduce a given shape using the mean shape and variation modes by the SSM instantiation equation:

$$
S=\bar{M}+\sum_{i=1}^{N} \omega_{i} D_{i}
$$

where $S$ is an instance of the SSM, $\bar{M}$ is the mean shape, $D_{i}$ is the $i^{\text {th }}$ variation mode, $N$ is the number of variation modes to be used, and $\omega_{i}$ is a weighting parameter for SSM instantiation. In our experiments, we used 15 most dominant variation modes of the SSM.

\subsection{Multi-slice to Volume Registration}

In our previous work, ${ }^{9}$ we performed a rigid registration between the mean shape of the SSM and the US data. The rigid registration was then followed by a deformable registration using 15 SSM weighting parameters. Since the shape of the pelvis in the SSM mean shape is different from the US data, the result of the rigid registration may not be accurate enough and can result in ultimately higher SSM to US registration errors. As a result, here, we improve our registration method by performing a simultaneous optimization of the rigid and shape parameters in each iteration of the optimizer. In this simultaneous optimization method, the rigid parameters that are initialized to the result of SSM mean shape to US rigid registration, are corrected in each iteration of the optimizer using the current SSM instance. The registration workflow is shown in Figure 1. The SSM and US are initialized to an overlapping position. In each iteration of the optimizer, the $15 \mathrm{SSM}$ weighting parameters, $\omega_{i}$, are updated and a new instance of the SSM is generated using Equation (1). The US tracking transformations are combined with the current rigid parameters of the registration, and slices are extracted from the SSM instance. An US image is then simulated from each of the extracted slices and the simulated US is compared with the corresponding US image using a correlation ratio-based similarity metric. During the registration process, the SSM variation mode weights and the rigid parameters are optimized simultaneously, until a SSM instance that best matches the shape of US image is achieved. The optimized rigid transformation corrects the US image alignment with this SSM instance. We use Linear Correlation of Linear Combination $\left(L C^{2}\right)$ as the similarity metric in our registration:

$$
L C^{2}=1-\frac{\sum(U S(x, y)-\operatorname{Sim} U S(x, y))^{2}}{N \times \operatorname{Var}(U S)}
$$

where $x$ and $y$ are the pixel index and $U S(x, y)$ and $\operatorname{Sim} U S(x, y)$ are the actual and simulated US image pixel intensities, respectively. $N$ is the number of overlapping pixels in US and simulated US images and $\operatorname{Var}(U S)$ is the variance of US image intensities. 


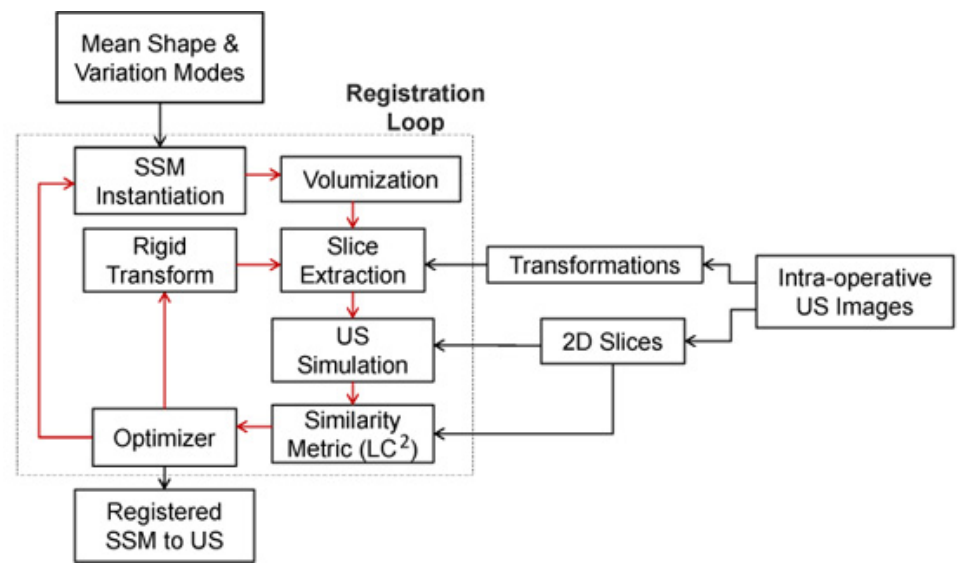

Figure 1. The workflow of the multi-slice to volume registration of the statistical shape model to intra-operative US. The 2D US images are obtained freehand and are optically tracked.

\subsection{Ultrasound Simulation}

The US simulation from the binary representation of the bone in the SSM is detailed in. ${ }^{9}$ We modify this method to improve the accuracy of the simulation. The bone surface in the US image is blurred due to the reflection of the US beam at the bone-soft tissue interface. Accordingly, we simulate the bone surface with a thickness of few pixels. In addition, the intensity of soft tissue pixels in the US are highly variable. As a result, we divide the soft tissue region of the SSM slice into subregions. The intensity of all the pixels in each subregion is set to the average intensity of corresponding pixels in the US. Figure 2 shows an US image, the corresponding slice extracted from the mean shape, and the US simulation with five subregions in the soft tissue region. As we increase the number of subregions, the accuracy of US simulation and registration increases as well. However, it is important to note that the intensity of each subregion in the simulated US slice is the average of the corresponding pixels in the actual US image. As a result, increasing the number of subregions exccessively increases the overall similarity of the US and simulated US images due to a closer intesity in the corresponding regions of the simulated and actual US slices, without necessarily having similar bone shapes. This can affect the optimization process and as a result, the number of subregions should be limited to an optimal number. Our experiments show that the optimal number of subregions is between 3 to 5 in each direction.
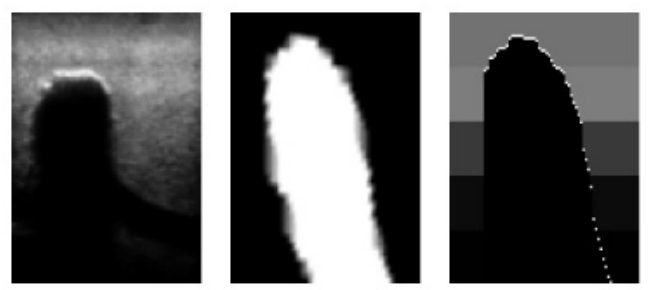

Figure 2. The simulated US slices of mean shape of the SSM; left: US slice of one dataset; middle: corresponding extracted slice of SSM mean shape; and right: simulated US from mean shape slice with five subregions in the soft tissue region. Note that the shape of the simulated US follows the shape of the bone in the SSM mean shape slice while the intensities are mean values calculated from the original US slice.

\section{EXPERIMENTS AND RESULTS}

To evaluate the accuracy of PaCS estimation with the proposed method, we construct five phantoms. One of the phantoms is made of a dry bone (Sawbones, Pacific Research Laboratories, Inc., Vashon, WA, USA) and the other four phantoms are built by rapid prototyping from CT data of four male patients. The CT data is collected at Kingston General Hospital, Kingston, ON from patients who provided consent to participate in this 
study. The printed 3D model is placed firmly in a plastic container so that the regions containing the anatomical landmarks are positioned facing the top. The model is then covered with an agar-based gel. ${ }^{11}$ The gel shows the same characteristics and speckle pattern as human soft-tissue in US images. For each phantom, 10 CT-visible fiducial markers (Beekley Corp., Bristol, CT, USA) are placed on two sides of the container.

\subsection{Data Acquisition}

For each phantom, three US sweeps are acquired: one on the pubic bone and one on each of the iliac crests. We use an L14-5/38 linear-array transducer and a Sonix RP US machine (Ultrasonix Medical Corp., Richmond, BC, Canada). The US frames are collected at a depth of $5 \mathrm{~cm}$ with probe frequency of $10 \mathrm{MHz}$. The probe is tracked using a dynamic reference body (DRB) (Traxtal Technologies Inc., Toronto, Canada) attached firmly to it and an Optotrack Certus System (Northern Digital Inc., Waterloo, ON, Canada). The US probe is calibrated to the tracking system using an $\mathrm{N}$-wire phantom in a water bath. ${ }^{12}$ The position of fiducial markers on the phantom container in the physical space of the tracker system are collected by a calibrated stylus pointer. The phantoms are CT scanned at high resolution $(0.69 \mathrm{~mm} \times 0.69 \mathrm{~mm} \times 0.62 \mathrm{~mm})$ using a GE Light-Speed series scanner (GE Healthcare, Waukesha, USA) for validation purposes. Fiducial markers are segmented manually in the CT and are registered to the fiducials in the US coordinate system using Horn's point-based registration method. ${ }^{13}$ The fiducial registration error (FRE) is $2.5,3.4,1.4,1.6$ and $1.2 \mathrm{~mm}$ for phantoms $1,2,3,4$, and 5 , respectively. The two sources of FRE are the segmentation error of the fiducial points in the CT, and the error in collecting fiducial coordinates in the US space using the stylus pointer.

\subsection{Results and Discussion}

We have perforemed two sets of experiments, in order to evaluate our registration method, and to demonstrate the importance of including US images of the pubic symphysis on precise localization of PaCS. In the first set of experiments, the SSM is registered to the collected US images from the three regions of intrest containing the four anatomical landmarks on the pelvis. The SSM to US registration using about 300 US images of the pelvis took less than 3 hours for all the test subjects. Figure 3 shows the slice overlay of the SSM mean shape (prior to registration) and registered SSM instance with the US. It can be seen that the proposed method can register the SSM slices to the US data effectively. Both the shape of the bone in the SSM and its position are corrected during the registration with the guidance of US data, and the patient's anatomy is retrieved. The registered SSM instance is then compared to the registered CT to evaluate the accuracy of the registration. A mean surface error is used to report the error which is calculated by averaging the distance of a set of points on the surface of the SSM to the closest surface points of the CT. The results of the registration are shown in Table 1. The overall registration error is high due to a lack of anatomical information in the posterior regions of the pelvis where an US scan is not available. However, in the ROI (where the US images are acquired) registration error is low as expected. An explanation for the registration error is that the SSM changes shape in a symmetrical fashion; as a result, the asymmetry of the patient's pelvis can cause registration error. An accurate registration of ROI is important because it is where the anatomical landmarks which form PaCS are located.

The four anatomical landmarks are picked on the registered SSM manually and the PaCS is calculated. The estimated PaCS is then compared to the ground truth PaCS in the registered CT data. The error of PaCS estimation is reported in Table 2. The translation error is calculated by the distance of the two origins and the rotation error is calculated by the angle between each axis in the two PaCS. As can be seen from Table 2, the PaCS of a patient can be estimated with an acceptable accuracy from the registered SSM instance to the US. The low rotation error in all experiments indicates that the result of registration can be a good replacement for pre-operative imaging in THR for localizing the PaCS. The PaCS localization error is partly caused by the US calibration error and the registration error of SSM to US. Additionally, the PaCS estimated on the registered SSM to US is compared to the ground truth PaCS, which is localized on the CT. Therefore, the inconsistency of the manual selection of the four anatomical landmarks on the CT and on the registered SSM can influence the evaluation of the PaCS estimation accuracy. As can be seen in the PaCS estimation error, the translation error in the $\mathrm{Z}$ direction is fairly high. The $\mathrm{Z}$ direction is almost parallel to the US beam propagation direction. In the US images, the total reflection at the bone edge makes the bone edge blurry which results in the higher translation error in this direction.

Proc. of SPIE Vol. 7964 79642U-4 


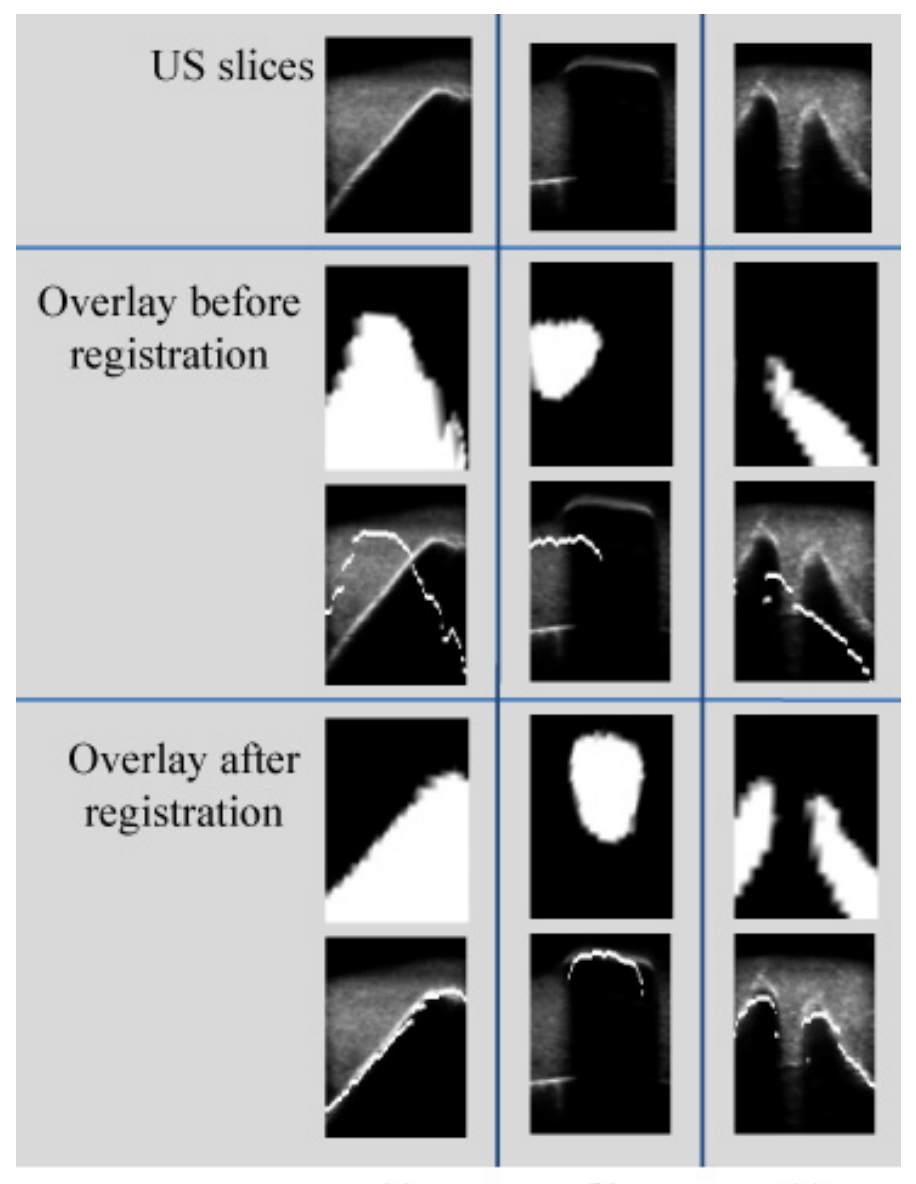

(a)

(b)

(c)

Figure 3. The US slice, extracted SSM slice and their overlay before and after the SSM to US registration for phantom 2. First row shows the US slices acquired from the ROI (where the anatomical landmarks are located). The two middle rows show the extracted slice from the mean shape before registration and its overlay with the US slice. The two bottom rows show the extracted SSM slice after registration and its overlay with the US slice. (a) The US acquired from the right iliac crest; (b) The US acquired from the pubic symphysis; (c) The US acquired from the left iliac crest.

Table 1. Mean surface error of the SSM to US registration compared to the CT of the phantoms.

\begin{tabular}{c|c|c} 
Dataset & $\begin{array}{c}\text { Mean surface error (Total volume) } \\
\text { mean } \pm \text { std }(\mathbf{m m})\end{array}$ & $\begin{array}{c}\text { Mean surface error (ROI) } \\
\text { mean } \pm \text { std } \mathbf{~ ( m m ) ~}\end{array}$ \\
\hline Phantom 1 & $5.66 \pm 4.57$ & $2.85 \pm 1.54$ \\
\hline Phantom 2 & $4.80 \pm 3.23$ & $2.34 \pm 1.47$ \\
\hline Phantom 3 & $5.58 \pm 4.16$ & $3.47 \pm 0.97$ \\
\hline Phantom 4 & $5.37 \pm 3.80$ & $2.63 \pm 0.76$ \\
\hline Phantom 5 & $4.36 \pm 3.66$ & $2.14 \pm 0.88$
\end{tabular}

To evaluate the sensitivity of our method to initialization of the parameters, we perform 25 SSM to US registration tests on each data set with different initializations. First, we rigidly register the SSM mean shape to the US. We then perturb this alignment by a uniformly distributed transformation of $\pm 5 \mathrm{~mm}$ in translation and \pm 5 degrees in rotation along each axis with the initial registration error of less than $12 \mathrm{~mm}$, and perform the SSM to the US registration. The initial registration error is calculated as the average distance of each corner of the perturbed volume to its coordinates in the initial position. The results of the registrations are compared with 
Table 2. The error of estimated PaCS of the registered SSM to US.

\begin{tabular}{c|c|c|c|c|c|c} 
& \multicolumn{2}{|c|}{$\begin{array}{c}\text { translation } \\
\text { error }(\mathbf{m m})\end{array}$} & \multicolumn{3}{c}{$\begin{array}{c}\text { rotation } \\
\text { error (degree) }\end{array}$} \\
\cline { 2 - 7 } Dataset & $\mathrm{x}$ & $\mathrm{y}$ & $\mathrm{z}$ & $\alpha$ & $\beta$ & $\gamma$ \\
\hline Phantom 1 & 0.46 & 1.10 & 3.46 & 0.20 & 2.05 & 1.99 \\
\hline Phantom 2 & 0.84 & 2.05 & 3.41 & 0.17 & 1.82 & 1.81 \\
\hline Phantom 3 & 0.64 & 3.80 & 2.28 & 0.17 & 1.84 & 1.83 \\
\hline Phantom 4 & 0.31 & 0.98 & 2.34 & 0.32 & 0.64 & 1.67 \\
\hline Phantom 5 & 0.99 & 1.57 & 2.36 & 0.45 & 0.36 & 1.36
\end{tabular}

the registered SSM to US with the accurate initialization, to calculate the final registration error. The results are shown in Table 3. It can be seen from this table that with initial TREs of less than $10 \mathrm{~mm}$, the registration technique is almost guaranteed to converge. The low mean and the standard deviation of final registration error indicates that the proposed registration algorithm is robust to the initialization parameters. It is important to note that the registration capture range is limited by the initial translation and rotation error, since higher error can reduce the initial overlap of mean shape slices and US images. In the case of poor initialization where no bone is present in the $37 \mathrm{~mm} \times 37 \mathrm{~mm}$ slices extracted from the mean shape, the optimizer may fall in a local minimum which causes the registration to fail.

Table 3. The final registration error for 25 multi-slice to volume registrations of statistical shape model to US with different initializations.

\begin{tabular}{c|c|c} 
Dataset & $\begin{array}{c}\text { Mean final } \\
\text { registration error } \pm \text { std }(\mathbf{m m})\end{array}$ & $\begin{array}{c}\text { Max initial } \\
\text { registration error }\end{array}$ \\
\hline Phantom 1 & $2.31 \pm 0.25$ & 10.30 \\
\hline Phantom 2 & $1.28 \pm 0.61$ & 11.92 \\
\hline Phantom 3 & $2.49 \pm 0.17$ & 9.24 \\
\hline Phantom 4 & $1.49 \pm 0.43$ & 11.92 \\
\hline Phantom 5 & $1.89 \pm 0.77$ & 9.67
\end{tabular}

In the second set of experiments, the US slices from the pubic symphysis are excluded from the image and the SSM is registered to the US using our proposed method. The PaCS is calculated on the registered SSM and the estimation error is calculated by comparison to the ground truth PaCS in the registered CT data. The results are reported in Table 4. A comparison of the results with the errors reported in Table 2 shows a significant increase in the rotation error along the $\mathrm{y}$ and $\mathrm{z}$ directions as expected. The reason for the increase in the error lies in the fact that the anatomical infromation from the phantom's pubic symphysis has been excluded. This lack of anatomical information results in an inaccurate registration of SSM to US in the pubis and as a result, an inaccurate estimation of the anterior pelvic plane. Accurate estimation of the anterior pelvic plane is critical in the measurements during THR as well as, the estimation of the $y$ and $z$ axis directions of the PaCS. ${ }^{4}$ Since the origin of the PaCS and the direction of $\mathrm{x}$ axis are estimated only based on the iliac crest landmarks, the translation error and the rotation error of $\mathrm{x}$ axis are not affected by the exclusion of the pubic US scans. The results of our cadaver study, ${ }^{9}$ in which the US data from the pubic symphysis was missing, are in accordance with our current results. These results demonstrate clearly the importance of including the pubic symphysis landmarks in the US data in order to have a precise estimation of the PaCS.

\section{CONCLUSION AND FUTURE WORK}

In this work, a phantom validation of multi-slice to volume registration between US data and an SSM of the pelvis is performed on five patient-based phantoms. Results show that the SSM can be registered to the US 
Table 4. The error of estimated PaCS of the registered SSM to US excluding the US slices from the pubic symphysis.

\begin{tabular}{c|c|c|c|c|c|c} 
& \multicolumn{2}{|c|}{$\begin{array}{c}\text { translation } \\
\text { error (mm) }\end{array}$} & \multicolumn{3}{c}{$\begin{array}{c}\text { rotation } \\
\text { error (degree) }\end{array}$} \\
\cline { 2 - 7 } Dataset & $\mathrm{x}$ & $\mathrm{y}$ & $\mathrm{z}$ & $\alpha$ & $\beta$ & $\gamma$ \\
\hline Phantom 1 & 0.73 & 1.59 & 3.23 & 0.47 & 3.05 & 5.82 \\
\hline Phantom 2 & 0.86 & 1.08 & 2.85 & 0.79 & 3.54 & 4.99 \\
\hline Phantom 3 & 0.86 & 2.32 & 2.01 & 1.37 & 4.67 & 4.04 \\
\hline Phantom 4 & 0.43 & 2.24 & 1.42 & 1.40 & 3.56 & 5.95 \\
\hline Phantom 5 & 1.03 & 1.15 & 1.81 & 0.92 & 3.67 & 3.85
\end{tabular}

using our registration method without any need for pre-processing the US data. The registered SSM has the potential to replace pre-operative imaging in THR and as a result, the patient's exposure to ionizing radiation can be eliminated altogether. The PaCS can be localized precisely using this radiation-free navigation system. The results show that the proposed registration method is robust to initialization error of up to $10 \mathrm{~mm}$. Precise registration of the landmarks in the pubic symphysis provides us with an accurate estmation of the anterior pelvic plane and the PaCS axis orientation.

Despite the validation presented here, further validation in a clinical setting is essential. To make the registration suitable for real-time performance in a clinical setting, acceleration of computation, potentially on a GPU hardware, must be acheievd. The nature of the US simulation lends itself well to the parallel processing available on a GPU ${ }^{14}$ and will be a source of significant reduction in computation time.

\section{ACKNOWLEDGMENTS}

This research was supported in part by Canadian Institutes of Health Research (CIHR) and Natural Sciences and Engineering Research Council of Canada (NSERC). We would like to also thank Prof. Russel Taylor and Dr. Ofri Sadowski, Ms. Gouthami Chintalapani and Mr. Pezhman Foroughi of the Johns Hopkins University for providing us with the statistical shape model data.

\section{REFERENCES}

1. R. Biedermann, A. Tonin, M. Krismer, F. Rachbauer, G. Eibl, and B. Stockl, "Reducing the risk of dislocation after total hip arthroplasty: The effect of orientation of the acetabular component," Journal of Bone and Joint Surgery. British volume 87-B(6), pp. 762-769, 2005.

2. B. Kavanagh, D. Ilstrup, and R. Fitzgerald, "Revision total hip arthroplasty," Journal of Bone and Joint Surgery 67(4), pp. 517-526, 1985.

3. J. Beckmann, C. Luring, M. Tingart, S. Anders, J. Grifka, and F. Kock, "Cup positioning in THA: current status and pitfalls. A systematic evaluation of the literature," Archives of Orthopaedic and Trauma Surgery 129(7), pp. 863-872, 2009.

4. C. Nikou, B. Jaramaz, A. M. DiGioia, and T. J. Levison, "Description of anatomic coordinate systems and rationale for use in an image-guided total hip replacement system," In Proceedings of Medical Image Computing and Computer-Assisted Intervention 1935, pp. 9-12, 2000.

5. C. S. K. Chan, P. J. Edwards, and D. J. Hawkes, "Integration of ultrasound-based registration with statistical shape models for computer-assisted orthopaedic surgery," In Proceedings of SPIE Medical Imaging 5032(1), pp. 414-424, 2003.

6. P. Foroughi, D. Song, G. Chintalapani, R. Taylor, and G. Fichtinger, "Localization of pelvic anatomical coordinate system using US/atlas registration for total hip replacement," In Proceedings of Medical Image Computing and Computer-Assisted Intervention 5242, pp. 871-879, 2008.

7. D. C. Barratt, C. S. Chan, P. J. Edwards, G. P. Penney, M. Slomczykowski, T. J. Carter, and D. J. Hawkes, "Instantiation and registration of statistical shape models of the femur and pelvis using 3D ultrasound imaging," Medical Image Analysis 12(3), pp. 358 - 374, 2008. 
8. S. Schumann, M. Puls, T. Ecker, K.-A. Siebenrock, and G. Zheng, "Determination of pelvic orientation from ultrasound images using patch-SSMs and a hierarchical speed of sound compensation strategy," Information Processing in Computer-Assisted Interventions, pp. 160-170, 2010.

9. S. Ghanavati, P. Mousavi, G. Fichtinger, P. Foroughi, and P. Abolmaesumi, "Multi-slice to volume registration of ultrasound data to a statistical atlas of human pelvis," In Proceedings of SPIE Medical Imaging 7625(1), pp. 76250O1-10, 2010.

10. G. Chintalapani, L. Ellingsen, O. Sadowsky, J. Prince, and R. Taylor, "Statistical atlases of bone anatomy: Construction, iterative improvement and validation," In Proceedings of Medical Image Computing and Computer-Assisted Intervention, pp. 499-506, 2007.

11. E. L. Madsen, M. A. Hobson, H. Shi, T. Varghese, and G. R. Frank, "Tissue-mimicking agar/gelatin materials for use in heterogeneous elastography phantoms," Physics in Medicine and Biology 50(23), pp. 5597$5618,2005$.

12. T. K. Chen, A. D. Thurston, M. H. Moghari, R. E. Ellis, and P. Abolmaesumi, "A real-time ultrasound calibration system with automatic accuracy control and incorporation of ultrasound section thickness," In Proceedings of SPIE Medical Imaging 6918(1), pp. 69182A1-11, 2008.

13. K. S. Arun, T. S. Huang, and S. D. Blostein, "Least-squares fitting of two 3-D point sets," IEEE Transactions on Pattern Analysis and Machine Intelligence 9(5), pp. 698-700, 1987.

14. O. Kutter, R. Shams, and N. Navab, "Visualization and GPU-accelerated simulation of medical ultrasound from CT images," Computer Methods and Programs in Biomedicine 94(3), pp. 250-266, 2009. 